\begin{tabular}{|c|l|}
\hline Title & Characterization of Hermitian symmetric spaces by fundamental forms \\
\hline Author(s) & Hwang, J. M; Y amaguchi, K. \\
\hline Citation & Hokkaido University Preprint Series in Mathematics, 569, 1-10 \\
\hline Issue Date & 2002-11 \\
\hline DOI & 10.14943/83714 \\
\hline Doc URL & http://hdl.handle.net/2115/69318 \\
\hline Type & bulletin (article) \\
\hline File Information & pre569.pdf \\
\hline
\end{tabular}

Instructions for use 
Characterization of Hermitian symmetric spaces by fundamental forms

Jun-Muk Hwang and Keizo Yamaguchi

Series \#569. November 2002 


\section{HOKKAIDO UNIVERSITY PREPRINT SERIES IN MATHEMATICS}

\#543 K. Sugano, On H-separable extensions of QF-3 rings, 7pages. 2001.

\#544 A. Arai, Non-relativistic limit of a Dirac-Maxwell operator in relativistic quantum elcctrodynamics, 27pages. 2001.

\#545 O. Sawada, On time-local solvability of the Navier-Stokes equations in Besov spaces, 30 pages. 2001.

\#546 C. M. Elliott, Y. Giga, and S. Goto, Dynamic boundary conditions for Hamilton-Jacobi equations, 27 pages. 2001.

\#547 Y. Nakano, Minimizing cohcrent risk measures of shortfall in discrete-time models with cone constraints, 22 pages. 2002.

\#548 K. tachizawa, A generalization of the Lieb-Thirring inequalities in low dimensions, 13 pages. 2002.

\#549 T. Nakazi, Absolute values and real parts for functions in the Smirnov class, 8 pages. 2002.

\#550 T. Nakazi and T. Watanabe, Properties of a Rubin's orthogonal function which is a linear combination of two inner functions, 9 pages. 2002.

\#551 T. Ohtsuka, A level set method for spiral crystal growth, 24 pages. 2002.

\#552 M.-H. Giga and Y. Giga, Minimal vertical singular diffusion preventing overturning for the Burgers equation, 18 pages. 2002 .

\#553 Y. Giga and P. Rybka, Berg's effect, 12 pages. 2002.

\#554 Y. Tonegawa, Domain clependent monotonicity formula for a singular perturlation problem, 14 pages. 2002 .

\#555 S. Izumiya and N. Takeuchi, New special curves and dovelopable surfaces, 10 pages. 2002.

\#556 S. Izumiya, K. Saji and N. Takeuchi, Singularities of line congruences, 17 pages. 2002.

\#557 S. Izumiya, D. Pei and M. C. Romero Fuster, The lightcone Gauss map of a spacelike surface in Minkowski 4-space, 21 pages. 2002.

\#558 S. Izumiya, D. Pei and M. C. Romero Fuster, Umbilicity of spacelike submanifolds of Minkowski space, 14 pages. 2002.

\#559 S. Izumiya, D. Pei and M. Takahashi, Curves and surfaces in Hyperbolic space, 16 pages. 2002.

\#560 J. Kato, On the uniqueness of nondecaying solutions for the Navier-Stokes equations, 19 pages. 2002.

\#561 M. Jinzenji and T. Sasaki, An Approach to $\mathcal{N}=4 A D E$ gauge Theory on K3, 29 pages. 2002

\#562 T.Nakazi and T.Yamamoto, Norms of some singular integral operators on weighted $L^{2}$ spaces, 27 pages.2002

\#563 A.Harris and Y.Tonegawa, A $\bar{\partial} \partial$-poincaré lemma for forms near an isolated complex singularity, 8 pages. 2002

\#564 M.Takahashi, Bifurcations of ordinary differential equations of Clairaut type, 23 pages.2002

\#565 G.Ishikawa, Classifying singular Legendre curves by contactomorphisms, 17 pages.2002

\#566 G.Ishikawa, Perturbations of Caustics and fronts, 17 pages.2002

\#567 Y.Giga, On regularizing-decay rate estmates for solutions to the Navier-Stokes initial value problem, 12 pages. 2002

\#568 T.Miyano, Strongry supercommuting serf-adjoint operators, 34 pages.2002 


\title{
Characterization of Hermitian symmetric spaces by fundamental forms
}

\author{
Jun-Muk Hwang and Keizo Yamaguchi
}

\section{Introduction}

In [L1] and [L2], Landsberg proved the following

Theorem A (Landsberg) Let $H$ be an irreducible compact Hermitian symmetric space of rank 2, different from the hyperquadric $\mathbf{Q}_{n} \subset \mathbf{P}_{n+1}$. Let $H \subset \mathbf{P}_{N}$ be a minimal non-degenerate equivariant embedding, equivalently, an embedding of $H$ in $\mathbf{P}_{N}$ defined by the complete linear system associated to the ample generator of $\operatorname{Pic}(H) \cong \mathrm{Z}$. Let $M \subset \mathbf{P}_{N}$ be a (not necessarily closed) complex sub-manifold with $\operatorname{dim}(M)=\operatorname{dim}(H)$ and $x \in M$ be a point in a neighborhood of which all the integer-valued differential invariants of $M$ remain constant. If the second fundamental form of $M$ at $x$ is isomorphic to the second fundamental form of $H$ at a point, then $M$ is projective-linearly equivalent to an open subset of $H$.

For the reducible case, he proved ([L1, Theorem 1])

Theorem $\mathbf{B}$ (Landsberg) Let $M \subset \mathbf{P}_{N}$ be a complex sub-manifold and $x \in M$ be a point in a neighborhood of which all the integer-valued differential invariants of $M$ remain constant. If the second fundamental form of $M$ at $x$ is isomorphic to the second fundamental form of a Segre variety $\mathbf{P}_{n_{1}} \times \mathbf{P}_{n_{2}} \subset \mathbf{P}_{\left(n_{1}+1\right)\left(n_{2}+1\right)-1}$, with $n_{1}, n_{2} \geq 2$, then $M$ is projective-linearly equivalent to an open subset of the Segre variety.

We refer the readers to [GH] and [L1] for historical back-ground on problems of this kind and the relation of such questions with other problems in projective algebraic geometry.

Our main result is a generalization of Theorem A to higher-order embeddings and to Hermitian symmetric spaces of higher rank. Precisely, we are going to prove

Theorem 1 Let $H$ be a compact Hermitian symmetric space which contains neither $\mathbf{P}_{n}, n \geq 1$ nor $\mathbf{Q}_{n}, n \geq 2$ as an irreducible factor. Let $H \subset \mathbf{P}_{N}$ be a non-degenerate equivariant embedding, or equivalently, the embedding by a complete linear system on $H$. Let $M \subset \mathbf{P}_{N}$ be a (not necessarily closed) complex sub-manifold with $\operatorname{dim}(M)=\operatorname{dim}(H)$ and $x \in M$ be a point in a neighborhood of which all the integer-valued differential invariants of $M$ remain constant. If the fundamental forms of $M$ at $x$ are isomorphic to the fundamental forms of $H$ at a point, then $M$ is projective-linearly equivalent to an open subset of $H$.

Note that for the minimal embedding of a Hermitian symmetric space of rank 2, the second osculating space spans the whole $\mathbf{P}_{N}$, so the higher fundamental forms are identically zero. Thus Theorem A is a special case of Theorem 1 .

It is well-known that the equivariant embeddings of $\mathbf{Q}_{n} \subset \mathbf{P}_{n+1}, n \geq 2$ and $\mathbf{P}_{n}, n \geq 1$ cannot be characterized by their fundamental forms. For example, for the standard embedding of $\mathbf{Q}_{n}$ in $\mathbf{P}_{n+1}$ and the second Veronese embedding of $\mathbf{P}_{n}$, one has to consider the cubic invariants in addition to the fundamental forms ([JM], [L1, Theorem 4]). However, even when $H$ contains $\mathbf{P}_{n}$ or $\mathbf{Q}_{n}$ as an irreducible factor, there are cases where our method can be applied. As an example, we will prove the following generalization of Theorem B: 
Theorem 2 Let $M \subset \mathbf{P}_{N}$ be a complex sub-manifold and $x \in M$ be a point in a neighborhood of which all the integer-valued differential invariants of $M$ remain constant. If the fundamental forms of $M$ at $x$ is isomorphic to those of a Segre variety $\mathbf{P}_{n_{1}} \times \cdots \times \mathbf{P}_{n_{m}} \subset \mathbf{P}_{N}, N=\prod_{i=1}^{m}\left(n_{i}+\right.$ 1) $-1, n_{i} \geq 2$, then $M$ is projective-linearly equivalent to an open subset of the Segre variety.

For the proof of Theorem 1 and Theorem 2, we use the theory of linear differential equations modelled on Hermitian symmetric spaces due to Y. Se-ashi [S]. This theory reduces the problem to the vanishing of certain Spencer cohomology groups by a reduction theory of principal bundles. In this sense, the proof is fundamentally different from [L1] and [L2], which depend on certain moving frame computation. Of course, one may say that the moving frame method and the reduction theory of principal bundles are just two different formulations of the same procedure. However, we believe that the more conceptual view-point of $[\mathrm{S}]$ is essential in the treatment of the higher rank and the higher order cases, because a simple-minded moving frame computation would get complicated heavily. We hope that presenting such a geometric application would make the deep work $[\mathrm{S}]$ more widely appreciated. See [SYY] for another application.

In Section 2, we will define the fundamental forms in the setting of [S]. In Section 3, an invariant-theoretic result concerning the fundamental forms of the Hermitian symmetric space is proved, which makes it possible to apply the result of $[S]$ in our problem. This reduces the problem to a calculation of Lic algebra cohomology. The computation of cohomology group will be dono using Kostant's harmonic theory in Scction 4. This computation had already appcared in $[\mathrm{SYY}]$ in the irreducible case.

\section{Fundamental forms as a system of linear differential equations}

We start with recalling some definitions from [S] (see also [SYY]). Let $M$ be a connected complex manifold. We denote by $T$ and $T^{*}$ the (holomorphic) tangent and the cotangent bundle of $M$ respectively. For a line bundle $E$ on $M$, we denote by $J^{p}(E)$ the bundle of $p$-jets of $E$. Its fiber at a point $x \in M$ is

$$
J_{x}^{p}(E):=\mathcal{O}_{x}(E) /\left(\mathcal{O}_{x}(E) \otimes \mathbf{m}_{x}^{p+1}\right) .
$$

Wo identify $J^{0}(E)=E$. For cach pair of non-negative integers $p \geq q$, we have a natural projection $\pi_{q}^{p}: J^{p}(E) \rightarrow J^{q}(E)$. For a section $s$ of $E$, denote its $p$-jet at $x$ by $j_{x}^{p}(s) \in J_{x}^{p}(E)$. Wo have a natural exact sequence of vector bundles

$$
0 \longrightarrow \operatorname{Sym}^{p} T^{*}(M) \otimes E \longrightarrow J^{p}(E) \stackrel{\pi_{p-1}^{p}}{\longrightarrow} J^{p-1}(E) \longrightarrow 0
$$

At cach $x \in M$, we have the induced exact sequence of vector spaces of the fibers at $x$

$$
0 \longrightarrow \operatorname{Sym}^{p} T_{x}^{*} \otimes E_{x} \longrightarrow J_{x}^{p}(E) \stackrel{\pi_{p-1}^{p}}{\longrightarrow} J_{x}^{p-1}(E) \longrightarrow 0 .
$$

A sub-bundle $R$ of $J^{p}(E)$ is called a system of linear differential equations of order $p$ on $E$. A section $s$ of $E$ over an open subset $U \subset M$ satisfying $j_{x}^{p}(s) \in R_{x}$ for each $x \in U$ is called a solution of $R$ over $U$. For each non-negative integer $r \leq p$, the subspace $\sigma_{x}^{r}(R) \subset \operatorname{Sym}^{r} T_{x}^{*}$ corresponding to $\pi_{r}^{p}\left(R_{x}\right) \cap\left(\operatorname{Sym}^{r} T_{x}^{*} \otimes E_{x}\right)$ under an identification $E_{x} \cong \mathbf{C}$ is called the $r$-th 
symbol of $R$ at $x$. It is independent of the choice of the identification $E_{x} \cong \mathbf{C}$. The direct sum $\sigma_{x}(R)=\bigoplus_{r=0}^{p} \sigma_{x}^{r}(R)$ is called the symbol of $R$ at $x$. The system $R \subset J^{p}(E)$ is said to be of finite type if $\sigma_{x}^{p}(R)=0$ for general $x \in X . R$ is said to be integrable if for each $\eta \in R_{x}$, there exists a solution $s$ over a neighborhood of $x$ for which $j_{x}^{p}(s)=\eta$. Such a solution is uniquely determined by $\eta$ when $R$ is of finite type.

Let $R$ (resp. $R^{\prime}$ ) be a system of order $p$ on a line bundle $E$ (resp. $E^{\prime}$ ) on a complex manifold $M$ (resp. $M^{\prime}$ ). We say that the symbol of $R$ at $x \in M$ is isomorphic to the symbol of $R^{\prime}$ at $y \in M^{\prime}$ if there exists a linear isomorphism $\theta: T_{x} \rightarrow T_{y}$ such that the induced linear isomorphism $\theta^{r}: \operatorname{Sym}^{r} T_{x}^{*} \rightarrow \operatorname{Sym}^{r} T_{y}^{*}$ sends $\sigma_{x}^{r}(R)$ to $\sigma_{y}^{r}\left(R^{\prime}\right)$ for each $0 \leq r \leq p$. We say that $R$ is locally equivalent to $R^{\prime}$ if there exists an open subset $U \subset M$ (resp. $U^{\prime} \subset M^{\prime}$ ) and a line bundle isomorphism $\varphi:\left.\left.E\right|_{U} \rightarrow E^{\prime}\right|_{U^{\prime}}$ such that the induced bundle isomorphism $J^{p}(\varphi):\left.\left.\left.J^{p}(E)\right|_{U} \rightarrow J^{p}\left(E^{\prime}\right)\right|_{U^{\prime}} \operatorname{maps} R\right|_{U}$ onto $\left.R^{\prime}\right|_{U^{\prime}}$.

Many interesting examples of integrable systems of linear differential equations of finite order arises from the projective differential geometry (e.g. [W]). In modern terms, it can be presented as follows. Let $M \subset \mathbf{P}_{N}$ be a non-degenerate complex sub-manifold. Let $E$ be the line bundle on $M$ defined by the restriction of the hyperplane line bundle of $\mathbf{P}_{N}$ and $\Sigma$ be the $(N+1)$ dimensional space of sections of $E$ coming from the sections of the hyperplane line bundle. Let $p>0$ be a positive integer such that an element of $\Sigma$ having a zero of order $\geq p$ at a point of $M$ is identically zero. Define $R_{x} \subset J_{x}^{p}(E)$ by

$$
R_{x}:=\left\{j_{x}^{p}(s) \mid s \in \Sigma\right\} .
$$

Then over a Zariski-open subset $M^{o}$ of $M$,

$$
R:=\bigcup_{x \in M^{\circ}} R_{x}
$$

defines a sub-bundle of $\left.J^{p}(E)\right|_{M^{\circ}}$, called the hyperplane system on $M$. By the construction, $R$ is of finite type and integrable. In fact,

$$
\pi_{q}^{p}\left(R_{x}\right)=\left\{j_{x}^{q}(s) \mid s \in \Sigma\right\} .
$$

Proposition 1 Let $M$ and $M^{\prime}$ be two non-degenerate sub-manifolds of $\mathbf{P}_{N}$. Let $R$ (resp. $\left.R^{\prime}\right)$ be the hyperplane system on $M$ (resp. $\left.M^{\prime}\right)$. Assume that $R$ and $R^{\prime}$ are of the same order. Then $R$ and $R^{\prime}$ are locally equivalent if and only if there exists a projective linear transformation sending an open subset of $M$ onto an open subset of $M^{\prime}$.

Proof. The 'if' part is trivial. Let us prove the 'only if' part. Let $E$ (resp. $E^{\prime}$ ) be the restriction of the hyperplane line bundle on $M$ (resp. $\left.M^{\prime}\right)$. Let $\Sigma$ (resp. $\Sigma^{\prime}$ ) be the sections of $E$ (resp. $E^{\prime}$ ) coming from the global sections of the hyperplane line bundle on $\mathbf{P}_{N}$. If $R$ and $R^{\prime}$ are locally equivalent, there exists an open subset $U \subset M$ (resp. $U^{\prime} \subset M^{\prime}$ ) and an isomorphism of line bundles $\varphi:\left.\left.E\right|_{U} \rightarrow E^{\prime}\right|_{U^{\prime}}$ mapping $\left.R\right|_{U}$ onto $\left.R^{\prime}\right|_{U^{\prime}}$. The solutions of $\left.R\right|_{U}$ are sent to the solutions of $\left.R^{\prime}\right|_{U^{\prime}}$. By the assumption that $R$ and $R^{\prime}$ are of finite type, cvery local solution of $R$ (resp. $R^{\prime}$ ) belongs to $\Sigma$ (resp. $\Sigma^{\prime}$ ). Thus $\varphi$ induces a lincar isomorphism from $\Sigma$ to $\Sigma^{\prime}$ which gives a projective linear transformation of $\mathbf{P}_{N}$ sending $U$ to $U^{\prime}$.

For $x \in M^{o}$, the symbol $\sigma_{x}^{r}(R) \subset \operatorname{Sym}^{r} T_{x}^{*}$ is called the $\mathbf{r}$-th fundamental form of $M$ at $x$ and is denoted by $F_{x}^{r}(M)$. We decree $F_{x}^{r}(M)=0$ for $r \geq p$. For two sub-manifolds $M, M^{\prime} \subset \mathbf{P}_{N}$, 
wo say that the fundamental forms of $M$ at $x \in M$ are isomorphic to the fundamental forms of $M^{\prime}$ at $y \in M^{\prime}$, if there exists a linear isomorphism $\theta: T_{x} \rightarrow T_{y}$ such that the induced linear map $\theta^{r}: \operatorname{Sym}^{r} T_{x}^{*} \rightarrow \operatorname{Sym}^{r} T_{y}^{*}$ sends $F_{x}^{r}(M)$ to $F_{y}^{r}\left(M^{\prime}\right)$ for each $r \geq 0$.

Remark Usually, the $r$-th fundamental form of $M$ at $x$ is defined as a linear map $\operatorname{Sym}^{r} T_{x} \rightarrow$ $N_{x}^{r}$ where $N_{x}^{r}$ is the $r$-th normal space at $x$ (e.g. $\left.[\mathrm{GH}]\right)$. Our $F_{x}^{r}(M) \subset \operatorname{Sym}^{r} T_{x}^{*}$ is the image of the dual of this linear map.

\section{Fundamental forms of Hermitian symmetric spaces}

We start with a few definitions regarding representations of abelian Lie algebras on graded vector spaces.

Let $W=\bigoplus_{r=0}^{p} W_{r}$ be a finite-dimensional graded vector space with $\operatorname{dim} W_{0}=1$. For each integer $k$, define

$$
\operatorname{gl}(W)_{k}:=\left\{X \in \operatorname{gl}(W) \mid X\left(W_{r}\right) \subset W_{k+r} \text { for all } r\right\} .
$$

Let $V$ be a finite dimensional vector space and $\mu: V \rightarrow \operatorname{gl}(W)$ be a representation of the abelian Lie algebra $V$ such that $\mu(V) \subset \operatorname{gl}(W)_{-1}$. Setting $\mathbf{p}_{-1}=\mu(V)$, define inductively for each $k \geq 0$,

$$
\mathbf{p}_{k}:=\left\{X \in \mathbf{g l}(W)_{k} \mid\left[\mathbf{p}_{-1}, X\right] \subset \mathbf{p}_{k-1}\right\} .
$$

Then the graded Lie subalgebra $\mathbf{p}=\bigoplus_{k=-1}^{\infty} \mathbf{p}_{k}$ of $\mathbf{g l}(W)$ is called the prolongation of $\mu$. For each non-negative integer $r$, define a homomorphism $\chi_{r}^{\mu}: W_{r} \rightarrow \operatorname{Sym}^{r} V^{*} \otimes W_{0}$ by

$$
\chi_{r}^{\mu}(w)\left(X_{1}, \ldots, X_{r}\right):=\mu\left(X_{1}\right) \cdots \mu\left(X_{r}\right)(w)
$$

for $w \in W_{r}$ and $X_{1}, \ldots, X_{r} \in V$. The right hand side is symmetric in $X_{1}, \ldots, X_{r}$ because $\mu$ is a representation of the abelian Lie algebra $V$. After fixing an identification $W_{0}=\mathbf{C}$, we denote by $\chi^{\mu}: W \rightarrow \bigoplus_{r=0}^{p} \operatorname{Sym}^{r} V^{*}$ the graded vector space homomorphism obtained as the sum of $\bigoplus_{r=0}^{p} \chi_{r}^{\mu}$. The homomorphism $\chi^{\mu}$ is called the symbol map of the representation $\mu: V \rightarrow \operatorname{gl}(W)_{-1}$. The following proposition is obvious.

Proposition 2 For $X \in V$, let $\iota(X): \operatorname{Sym}^{\bullet} V^{*} \rightarrow \operatorname{Sym}^{\bullet} V^{*}$ denote the inner multiplication by $X$. Then the image of $\chi^{\mu}$ is invariant under $\iota(X)$ for any $X \in V$ and

$$
\iota(X) \circ \chi^{\mu}(w)=\chi^{\mu}(\mu(X) \cdot w)
$$

for every $X \in V$ and $w \in W$.

Now let $\mathbf{l}=\mathbf{l}_{-1}+\mathbf{l}_{0}+\mathbf{l}_{1}$ be a semi-simple graded Lie algebra. Let $L$ be a connected Lie group with Lie algebra $\mathbf{l}$ and $L^{\prime}$ be a connected subgroup of $L$ with Lie algebra $\mathbf{l}^{\prime}=\mathbf{l}_{0}+\mathbf{l}_{1}$. Then the homogeneous space $L / L^{\prime}$ is a compact Hermitian symmetric space. Conversely, for any compact Hermitian symmetric space, let $\mathbf{I}$ be the Lie algebra of global holomorphic vector fields and $\mathbf{l}^{\prime}$ be the subalgebra consisting of vector fields vanishing at a base point. Then there exists a grading $\mathbf{l}=\mathbf{l}_{-1}+\mathbf{l}_{0}+\mathbf{l}_{1}$ such that $\mathbf{l}^{\prime}=\mathbf{l}_{0} \oplus \mathbf{l}_{1}$.

Given a non-degenerate equivariant embedding of the Hermitian symmetric space $L / L^{\prime} \subset \mathbf{P} S^{*}$ for a finite-dimensional vector space $S$, we get a faithful irreducible representation $\rho: 1 \rightarrow \operatorname{gl}(S)$ such that

$$
S_{0}:=\left\{s \in S \mid \rho\left(\mathbf{l}_{-1}\right)(s)=0\right\}
$$


is 1-dimensional. Define for $r \geq 0$,

$$
S_{r+1}:=\rho\left(\mathbf{l}_{1}\right)\left(S_{r}\right) .
$$

There exists $p>0$ such that $S_{r} \neq 0$ for $r=0,1, \ldots, p-1$ and $S_{r}=0$ for $r \geq p$. Moreover

$$
S=\bigoplus_{r=0}^{p} S_{r}
$$

Set $V:=1_{-1}$ and let $\nu: V \rightarrow \operatorname{gl}(S)$ be the representation obtained by restricting $\rho: 1 \rightarrow \operatorname{gl}(S)$ to $\mathbf{l}_{-1}$. Then $\nu: V \rightarrow \operatorname{gl}(S)_{-1}$ with respect to the grading $S=\bigoplus_{r=0}^{p} S_{r}$.

We recall the following result on the prolongation of $\nu$.

Proposition 3 ([S,Proposition 4.4.1], or [SYY, (2.5)] for the irreducible case) Regard 1 as a subalgebra of $\mathbf{g l}(S)$ by the given faithful irreducible representation $\rho: \mathbf{l} \rightarrow \mathbf{g l}(S)$. Let $\mathbf{z}$ be the centralizer of $\mathbf{l}$ in $\mathbf{g l}(S)$. For the representation $\nu: V:=\mathbf{l}_{-1} \rightarrow \operatorname{gl}(S)_{-1}$, the prolongation is $\mathbf{p}_{-1} \cong V$ (i.e. $\mu$ is injective), $\mathbf{p}_{0}=\mathbf{1}_{0}+\mathbf{z}, \mathbf{p}_{1}=\mathbf{1}_{1}$ and $\mathbf{p}_{k}=0$ for $k \geq 2$. In particular, $\mathbf{p}$ is a reductive graded Lie algebra whose semi-simple part is the graded semi-simple Lie algebra $\mathbf{l}$.

The following result on the symbol map of $\nu$ is contained in $[\mathrm{S}]$. Note that $\mathbf{l}_{-1}$ is naturally identified with the tangent space of the Hermitian symmetric space $L / L^{\prime}$ at the base point.

Proposition 4 Let $V=\mathbf{l}_{-1}$ and $\nu: V \rightarrow \operatorname{gl}(S)_{-1}$ be the representation obtained by restricting $\rho: \mathbf{l} \rightarrow \mathbf{g l}(S)$ with respect to the grading $S=\bigoplus_{r=0}^{p} S_{r}$. Then $\chi^{\nu}: \bigoplus_{r=0}^{p} S_{r} \rightarrow \bigoplus_{r=0}^{p} \mathrm{Sym}^{r} V^{*}$ is injective and the image $\chi_{r}^{\rho}\left(S_{r}\right) \subset \operatorname{Sym}^{r} V^{*}$ coincides with the $r$-th fundamental form $F_{y}^{r}\left(L / L^{\prime}\right) \subset$ $\operatorname{Sym}^{r}\left(\mathbf{l}_{-1}\right)^{*}$ of $L / L^{\prime} \subset \mathbf{P} S^{*}$.

Proof. The injectivity of $\chi_{r}^{\nu}$ is $\left[S\right.$, Proposition 4.3.1]. That the image $\chi_{r}^{\rho}\left(S_{r}\right) \subset \operatorname{Sym}^{r} V^{*}$ coincides with the $r$-th fundamental form is [S, Proposition 2.4.1.].

Now we regard $S=\bigoplus_{r=0}^{p} S_{r}$ as a graded vector subspace of $\bigoplus_{r=0}^{p} \operatorname{Sym}^{r} V^{*}$ by the injection $\chi^{\nu}$. By Proposition 2, the action of $V$ on $S$ by $\nu$ coincide with the restriction of the inner multiplication. The following invariant-theoretic result implies the 'rigidity' of the fundamental forms of Hermitian symmetric spaces.

Proposition 5 Regard $S=\bigoplus_{r=0}^{p} S_{r}$ as a graded subspace of $\oplus_{r=0}^{p} \operatorname{Sym}^{r} V^{*}$ as explained above. Let $P=\bigoplus_{r=0}^{p} P_{r}$ be a graded subspace of $\bigoplus_{r=0}^{p} \mathrm{Sym}^{r} V^{*}$ which has the same discrete invariants as $S$ under the action of $G L(V)$. Then $P$ is in the $G L(V)$-orbit of $S$. In other words, there exist finitely many $G L(V)$-invariant $\mathbf{Z}$-valued functions on the Grassmannian $G r\left(\operatorname{dim} S, \oplus_{r=0}^{p} \operatorname{Sym}^{r} V^{*}\right)$ of $(\operatorname{dim} S)$-dimensional subspaces in $\oplus_{r=0}^{p} \operatorname{Sym}^{r} V^{*}$, such that if all these functions have the same values on $P$ and $S$, then $P$ is in the orbit of $S$ under the $G L(V)$-action on the Grassmannian.

Proof. By Proposition 2, $S$ is invariant under $\iota(X)$ for all $X \in V$. Since $\operatorname{dim} \iota(X) P$ and $\operatorname{dim}(P \cap \iota(X) P)$ must be cqual to the corresponding dimensions for $S$, we conclude that $P$ is also invariant under $\iota(X)$. Moreover the action of $\iota(X)$ and $\iota(Y)$ on $P$ commute for any $X, Y \in V$. Thus we have a representation $\mu: V \rightarrow \operatorname{gl}(P)_{-1}$. The dimension of the prolongation of $\mu$ and the dimension of the prolongation of $\nu: V \rightarrow \operatorname{gl}(S)$ are equal by the assumption on $P$. So are the dimensions of their radicals. This implics, by Proposition 3, the prolongation $\mathbf{p}=\mathbf{p}_{-1}+\mathbf{p}_{0}+\mathbf{p}_{1}+\cdots$ of $\mu$ is reductive and isomorphic to $\mathbf{l}_{-1}+\left(\mathbf{l}_{0}+\mathbf{z}\right)+\mathbf{l}_{1}$. By the same reasoning; the action of the prolongation $\mathrm{p}$ on $P$ is isomorphic to the irreducible representation of 1 on $S$. In particular, $\mu: V \rightarrow \operatorname{gl}(P)_{-1}$ is isomorphic to $\nu: V \rightarrow \operatorname{gl}(S)_{-1}$ under a linear automorphism of 
$V$ and a graded vector space isomorphism $P \cong S$. Consider $\chi^{\mu}: P \rightarrow \bigoplus_{r=0}^{p} \operatorname{Sym}^{r} V^{*}$. The image $\chi^{\mu}(P) \subset \bigoplus_{r=0}^{p} \mathrm{Sym}^{r} V^{*}$ is completely determined by the structure of $P$ as a $V$-module. Thus $\operatorname{dim} \chi^{\mu}(P)=\operatorname{dim} \chi^{\nu}(S)$ and $\chi^{\mu}(P)$ is in the $G L(V)$-orbit of $\chi^{\nu}(S)$. But by our definition of $S$ as the subspace of $\oplus_{r=0}^{p} \operatorname{Sym}^{r} V^{*}, \chi^{\nu}(S)=S$. Since dim $\chi^{\mu}(P) \cap P$ is an integer-valued invariant of $P$, we conclude $P=\chi^{\mu}(P)$ is in the $G L(V)$-orbit of $\chi^{\nu}(S)=S$.

\section{Vanishing of Spencer cohomology}

Let $\mathbf{l}=\mathbf{l}_{-1}+\mathbf{l}_{0}+\mathbf{l}_{1}$ be a graded semi-simple Lie algebra associated to a Hermitian symmetric: space as explained in Section 3. We recall the description of the gradation of 1 in terms of the root system. Let $\mathbf{h} \subset \mathbf{l}_{0}$ be a Cartan subalgebra of $\mathbf{l}$. Then there exists a unique element $Z \in \mathbf{h}$, called the characteristic element, with the property that

$$
\mathbf{1}_{i}=\{X \in 1 \mid[Z, X]=i X\}
$$

for each $i=-1,0,1$. Choose a simple root system $\Delta=\left\{\alpha_{1}, \ldots, \alpha_{\ell}\right\}$ such that $\alpha_{i}(Z) \geq 0$ for all $i=1, \ldots, \ell$. Then $Z$ determines a partition $\Phi^{+}=\Phi_{0}^{+} \cup \Phi_{1}^{+}$of the set of positive roots by $\Phi_{k}^{+}=\left\{\alpha \in \Phi^{+} \mid \alpha(Z)=k\right\}$ for $k=0,1$, such that

$$
\begin{aligned}
\mathbf{l}_{-1} & =\bigoplus_{\alpha \in \Phi_{1}^{+}} \mathbf{g}_{-\alpha} \\
\mathbf{l}_{0} & =\mathbf{h} \oplus \bigoplus_{\alpha \in \Phi_{0}^{+}}\left(\mathbf{g}_{\alpha} \oplus \mathbf{g}_{-\alpha}\right) \\
\mathbf{l}_{1} & =\bigoplus_{\alpha \in \Phi_{1}^{+}} \mathbf{g}_{\alpha}
\end{aligned}
$$

where $\mathbf{g}_{\alpha}$ is the root space. Define

$$
\Delta_{1}=\left\{\alpha_{i_{1}}, \ldots, \alpha_{i_{m}}\right\}:=\{\alpha \in \Delta \mid \alpha(Z)=1\} .
$$

Recall

$$
<\beta, \gamma>=\frac{2(\beta, \gamma)}{(\gamma, \gamma)}
$$

for $\beta, \gamma \in \mathbf{h}^{*}$. Let $\lambda_{1}, \ldots, \lambda_{\ell}$ be the fundamental weights and $h_{\alpha_{1}}, \ldots, h_{\alpha_{\ell}}$ be the co-roots, namely,

$$
<\lambda_{i}, \alpha_{j}>=\delta_{i j}, \quad \beta\left(h_{\alpha_{i}}\right)=<\beta, \alpha_{i}>\quad \text { for all } \beta \in \mathbf{h}^{*} .
$$

By a direct computation, we have

Lemma 1 Suppose $\lambda_{i_{1}}+\cdots+\lambda_{i_{m}}=\sum_{i=1}^{\ell} a_{i} \alpha_{i}$. Then $Z=\sum_{i=1}^{\ell} a_{i} h_{\alpha_{i}}$.

Let $W$ be the Weyl group of the root system $\Phi$. For an element $\sigma \in W$, put $\Phi_{\sigma}=\sigma\left(-\Phi^{+}\right) \cap$ $\Phi^{+}$. Define

$$
\begin{aligned}
W^{0} & :=\left\{\sigma \in W \mid \Phi_{\sigma} \subset \Phi_{1}^{+}\right\} \\
W^{0}(1) & :=\left\{\sigma \in W^{0} \mid \leftleftarrows\left(\Phi_{\sigma}\right)=1\right\}
\end{aligned}
$$

where $\leftleftarrows\left(\Phi_{\sigma}\right)$ is the number of roots in $\Phi_{\sigma}$. For $\alpha_{i} \in \Delta$. let $\sigma_{i} \in W$ be the corresponding reflection. 
Lemma $2 W^{0}(1)=\left\{\sigma_{\alpha} \mid \alpha \in \Delta_{1}\right\}=\left\{\sigma_{i_{1}}, \ldots, \sigma_{i_{m}}\right\}$.

Proof. The inclusion $\left\{\sigma_{i_{1}}, \ldots, \sigma_{i_{m}}\right\} \subset W^{0}(1)$ is immediate. By [Hu, 10.3 Lemma A], elements of $W^{0}(1)$ are simple reflections. If $\sigma_{i} \in W^{0}(1)$, then $\Phi_{\sigma_{i}}=\left\{\sigma_{i}\right\}$ and $\Phi_{\sigma} \subset \Phi_{1}^{+}$. Thus $\alpha_{i} \in \Delta_{1}$. $\square$

For a finite-dimensional 1-module $\Gamma$ for which the weights of $Z$ are integers, let $\Gamma_{p}$ be the subspace of $\Gamma$ consisting of eigenvectors of $Z$ with eigenvalue $p \in \mathbf{Z}$. Set $C^{p, q}:=\Lambda^{q}\left(\mathbf{1}_{-1}\right)^{*} \otimes \Gamma_{p-1}$ and define $\partial: C^{p, 1} \rightarrow C^{p-1,2}$ by

$$
\partial c\left(X_{0}, X_{1}\right)=X_{0} \cdot c\left(X_{1}\right)-X_{1} \cdot c\left(X_{0}\right)
$$

for $c \in C^{p, 1}=\left(\mathbf{1}_{-1}\right)^{*} \otimes \Gamma_{p-1}$ and $\partial: C^{p+1,0} \rightarrow C^{p, 1}$ by

$$
\partial c(X)=X \cdot c
$$

for $x \in \Gamma_{p}$. For each integer $p$, the $p$-th Spencer cohomology group associated to $\Gamma$ is defined by

$$
H^{p, 1}\left(\mathbf{1}_{-1}, \Gamma\right):=\frac{\left(\operatorname{Ker} \partial: C^{p, 1} \longrightarrow C^{p-1,2}\right)}{\left(\operatorname{Im} \partial: C^{p+1,0} \longrightarrow C^{p, 1}\right)}
$$

They give the decomposition of the Lie algebra cohomology group

$$
H^{1}\left(\mathbf{1}_{-1}, \Gamma\right)=\oplus_{p} H^{p, 1}\left(\mathbf{1}_{-1}, \Gamma\right)
$$

which is just the $Z$-eigenspace decomposition.

Given a faithful irreducible l-module $S$ with $\operatorname{dim} S_{0}=1, \operatorname{g} \subset \operatorname{gl}(S)$ be the prolongation of $1_{-1}$-action on $S$ as defined in the last section. Let $\mathbf{g}^{\perp} \subset \operatorname{gl}(S)$ be the orthogonal complement with respect to the Killing form on $\operatorname{gl}(S)$. Then $\mathrm{g}^{\perp}$ is an l-module where the weights of $Z$ are integers. So $H^{p, 1}\left(\mathbf{l}_{-1}, \mathbf{g}^{\perp}\right)$ makes sense.

Let $R$ be a system of linear differential equations of order $p$ on a complex manifold $M$. Given a graded subspace $S=\bigoplus_{i=0}^{p} S_{i} \subset \operatorname{Sym}^{\bullet} V$ for a vector space $V, \operatorname{dim} V=\operatorname{dim} M$, we say that the symbol $\sigma_{x}(R)$ is isomorphic to $S$ if there exists a linear isomorphism $\psi: T_{x}^{*} \rightarrow V$ such that the induced graded linear isomorphism $\operatorname{Sym}^{\bullet} T_{x}^{*} \rightarrow \operatorname{Sym}^{\bullet} V$ sends $\sigma_{x}(R)$ onto $S$.

We will reduce the proof of Theorem 1 and Theorem 2 to the vanishing of certain Spencer cohomology groups by using the following result of Y. Se-ashi. Theorem 3 appeared as Theorem 5.1.2, Theorem 5.2.2 and Theorem 5.3.1 in [S]. We recommend [SYY, 2.4] for an over-view of its proof, where it appeared as 'Theorem A'.

Theorem 3 (Se-ashi) Let $H \subset \mathbf{P}_{N}$ be an equivariantly embedded Hermitian symmetric space and $F=\oplus_{r=0}^{p} F^{r}$ be the fundamental forms at a point of $H$. Assume that $H^{p, 1}\left(\mathbf{1}_{-1}, \mathbf{g}^{\perp}\right)=0$ for all $p \geq 1$. Suppose a system $R$ on a line bundle $E$ of a complex manifold $M$ has its symbol at each point $x \in M$ isomorphic to $F$. Then $R$ is locally equivalent to the hyperplane system on $H \subset \mathbf{P}_{N}$.

For the computation of the Spencer cohomology we will use Kostant's result. The following is a translation of [Ko, Theorem 5.14] into our situation (cf. [SYY,2.j Theorem B]). See also $[Y, 5.1]$ for a stream-lined review of Kostant theory. Note that $\mathbf{l}_{1}=\left(\mathbf{l}_{-1}\right)^{*}$ by the Killing form. 
Theorem 4 (Kostant) Let $\Gamma$ be an irreducible 1 -module and $\gamma$ be its lowest weight. Then we have an $\mathbf{l}_{0}$-module decomposition

$$
H^{1}\left(\mathbf{1}_{-1}, \Gamma\right)=\bigoplus_{\sigma \in W^{0}(1)} \mathcal{H}^{\xi_{\sigma}}
$$

where $\mathcal{H}^{\xi_{\sigma}}$ is the irreducible $\mathbf{l}_{0}$-module with the lowest weight $\xi_{\sigma}:=\sigma(\gamma)+\alpha$ for the simple root $\alpha$ satisfying $\sigma=\sigma_{\alpha}$.

This has the following consequence for the Spencer cohomology groups.

Proposition 6 Let $Z \in \mathbf{h}$ be the characteristic element of the graded Lie algebra $\mathbf{1}$. Let $\Gamma$ be an irreducible 1-module such that the weights of $Z$ on $\Gamma$ are integers. Let $\gamma$ be the lowest weight of $\Gamma$. Then $H^{p, 1}\left(1_{-1}, \Gamma\right) \neq 0$ only if $p-1=\sigma_{i_{k}}(\gamma)(Z)$ for some $i_{k}$.

Proof. $H^{p, 1}\left(\mathbf{l}_{-1}, \Gamma\right)$ is the cigenspace of $Z$ with eigenvalue $p$. By Theorem 4 , it is non-zero if there exists $\sigma_{i} \in W^{0}(1)$ such that

$$
p=\xi_{\sigma_{i}}(Z)=\sigma_{i}(\gamma)(Z)+\alpha_{i}(Z) .
$$

Thus the result follows from $W^{0}(1)=\left\{\sigma_{i_{1}}, \ldots, \sigma_{i_{m}}\right\}$ by Lemma 2 and $\alpha_{i_{k}}(Z)=1$.

The following is an honest generalization of [SYY, Theorem 2].

Proposition 7 Let Let $\mathbf{l}=\mathbf{l}_{-1}+\mathbf{l}_{0}+\mathbf{l}_{1}$ be the graded semi-simple Lie algebra associated to a Hermitian symmetric space which contains neither a projective space nor a hyperquadric as an irreducible factor. Then for any irreducible 1-module $\Gamma$,

$$
H^{p, 1}\left(\mathbf{1}_{-1}, \Gamma\right)=0 \text { for all } p \geq 1 \text {. }
$$

Proof.

$$
\begin{aligned}
\sigma_{1_{k}}(\gamma)(Z) & =\left(\gamma-<\gamma, \alpha_{i_{k}}>\alpha_{i_{k}}\right)(Z) \\
& =\gamma(Z)-<\gamma, \alpha_{i_{k}}>
\end{aligned}
$$

By Lemma 1,

$$
\sigma_{i_{k}}(\gamma)(Z)=\left(a_{i_{k}}-1\right)<\gamma, \alpha_{i_{k}}>+\sum_{i \neq i_{k}} a_{i}<\gamma, \alpha_{i}>
$$

Since $\gamma$ is the lowest weight, we have $\left\langle\gamma, \alpha_{i}\right\rangle \leq 0$ for $i=1, \ldots, \ell$ and $\left\langle\gamma, \alpha_{j}\right\rangle<0$ for some $j$. Since $a_{i}$ is an entry of the inverse matrix of the Cartan matrix, $a_{i}>0$. In particular, if $a_{i_{k}}>1$, then $\sigma_{i_{k}}(\gamma)(Z)<0$ for every $\gamma$. The only cases when $a_{i_{k}} \leq 1$ are the following (e.g. [Hu, 13.2 Table 1]).

- $a_{i_{k}}=\frac{\ell}{\ell+1}$ when $\alpha_{i_{k}}$ is the root $\alpha_{1}$ of $A_{\ell}, \ell \geq 1$.

- $a_{i_{k}}=1$ when $\alpha_{i_{k}}$ is the root $\alpha_{2}$ of $A_{3}$.

- $a_{i_{k}}=1$ when $\alpha_{i_{k}}$ is the root $\alpha_{1}$ of $B_{\ell}, \ell \geq 2$. 
- $a_{i_{k}}=1$ when $\alpha_{i_{k}}$ is the root $\alpha_{1}$ of $D_{\ell}, \ell \geq 4$.

In these cases, the corresponding irreducible factor of the Hermitian symmetric space is a projective space or a hyperquadric.

Proof of Theorem 1. Applying Proposition 7 to cach irreducible factor of $\mathbf{g}^{\perp}$, we see that $H^{p, 1}\left(\mathbf{l}_{-1}, \mathbf{g}^{\perp}\right)=0$ for all $p \geq 1$. By Proposition 5 , the fundamental forms of $M$ at cach point of a neighborhood of $x$ are isomorphic to the fundamental forms of $H$ at a point. Thus Theorem 1 follows from Theorem 3 and Proposition 1.

To prove Theorem 2, let us study the corresponding $\mathbf{l}$-modules. Let $\mathbf{l}=\mathbf{s} \mathbf{l}_{n_{1}+1} \oplus \cdots \oplus \mathbf{s}_{n_{m}+1}$ with $n_{i} \geq 2$ for all $i=1, \ldots, m$. Let us denote the simple roots of $\mathbf{s l}_{n_{j}+1}$ by $\left\{\alpha_{1}^{j}, \ldots, \alpha_{n_{j}}^{j}\right\}$ using the standard enumeration of simple roots of the simple Lie algebra of type $A$. For the Hermitian symmetric space $\mathbf{P}_{n_{1}} \times \cdots \times \mathbf{P}_{n_{m}}$, the corresponding gradation of 1 has

$$
\Delta_{1}=\left\{\alpha_{1}^{1}, \alpha_{1}^{2}, \ldots, \alpha_{1}^{m}\right\} .
$$

The fundamental weights of $\mathbf{1}$ are denoted by

$$
\lambda_{1}^{1}, \ldots, \lambda_{n_{1}}^{1}, \lambda_{1}^{2}, \ldots, \lambda_{n_{2}}^{2}, \ldots, \lambda_{1}^{m}, \ldots, \lambda_{n_{m}}^{m} .
$$

Let $U^{j}=\mathbf{C}^{n_{j}+1}$ be the basic representation space of $\mathbf{s l}_{n_{j}+1}$ corresponding to the fundamental woight $\lambda_{1}^{j}$. Let $S:=\left(U^{1}\right)^{*} \otimes \cdots \otimes\left(U^{m}\right)^{*}$ bo the basic representation space of $\mathbf{l}$. Using $\mathbf{g l}\left(\left(U^{i}\right)^{*}\right)=$ $\mathbf{C} \cdot \mathrm{Id}+\operatorname{sl}\left(\left(U^{i}\right)^{*}\right)$ and $\operatorname{sl}\left(\left(U^{i}\right)^{*}\right) \cong \mathbf{s l}\left(U^{i}\right)$, we have the l-module decomposition

$$
\operatorname{gl}(S)=\left(\mathbf{C} \cdot \operatorname{Id}+\operatorname{sl}\left(U^{1}\right) \oplus \cdots \oplus \operatorname{sl}\left(U^{m}\right)\right)+\mathbf{g}^{\perp}
$$

where $\mathrm{g}^{\perp}$ is a sum of tensor products of the form $\operatorname{sl}\left(U^{i_{1}}\right) \otimes \cdots \otimes \operatorname{sl}\left(U^{i_{k}}\right)$ for some $k \geq 2$. By Proposition 1, Proposition 5 and Theorem 3, the proof of Theorem 2 is reduced to the following.

Proposition 8 In the above notation, $H^{p, 1}\left(\mathbf{1}_{-1}, \mathbf{g}^{\perp}\right)=0$ for all $p \geq 1$.

Proof. Let $\gamma$ be the lowest weight of $\mathbf{s l}\left(U^{i_{1}}\right) \otimes \cdots \otimes \mathbf{s l}\left(U^{i_{k}}\right), k \geq 2$. By Proposition 6 , it suffices to show, for cach $\ell=1, \ldots, m$,

$$
\gamma(Z)-<\gamma, \alpha_{1}^{\ell}>\leq-1
$$

Since the adjoint representation $\mathbf{s l}\left(U^{j}\right)$ has highest weight $\lambda_{1}^{j}+\lambda_{n_{j}}^{j}$,

$$
\gamma=-\sum_{j=1}^{k}\left(\lambda_{1}^{i_{j}}+\lambda_{n_{i_{j}}}^{i_{j}}\right) .
$$

When $\lambda_{1}^{j}=\sum_{i=1}^{n_{j}} a_{i}^{j} \alpha_{i}^{j}$, Lemma 1 says

$$
Z=\sum_{j=1}^{m} \sum_{i=1}^{n_{j}} a_{i}^{j} h_{\alpha_{i}^{j}} .
$$

Lsing (c.g. from $\left[\mathrm{Hu}, 13.2\right.$ Table 1]) $a_{1}^{j}=\frac{n_{j}}{n_{j}+1}$ and $a_{n_{j}}^{j}=\frac{1}{n_{j}+1}$, we have

$$
\gamma(Z)=-\sum_{j=1}^{k}\left(a_{1}^{i_{j}}+a_{n_{i_{j}}}^{i_{j}}\right)=-k
$$


whilc

$$
\begin{gathered}
-<\gamma, \alpha_{1}^{\ell}>=<\lambda_{1}^{i_{j}}+\lambda_{n_{i_{j}}}^{i_{j}}, \alpha_{1}^{i_{j}}>=1 \text { when } \ell=i_{j} \text { and } \\
-<\gamma, \alpha_{1}^{\ell}>=0 \text { when } \ell \neq i_{1}, \ldots, i_{k} .
\end{gathered}
$$

Thus $\gamma(Z)-<\gamma, \alpha_{1}^{\ell}>\leq-1$.

Remark When $n_{1}=1$ and $2 \leq n_{2}, \ldots, n_{m}$, the adjoint representation $\mathbf{s l}\left(U^{1}\right)$ has highest weight $2 \lambda_{1}^{1}=\alpha_{1}^{1}$. Thus $\mathrm{g}^{\perp}$ has an irreducible factor with lowest weight

$$
\gamma=-2 \lambda_{1}-\lambda_{1}^{2}-\lambda_{n_{2}}^{2} .
$$

Then the same calculation as in the proof of Proposition 8 shows

$$
\begin{aligned}
\gamma(Z)= & -2 a_{1}^{1}-a_{1}^{2}-a_{n_{2}}^{2}=-2 \\
& -<\gamma, \alpha_{1}^{1}>=2 .
\end{aligned}
$$

Thus $H^{1,1}\left(\mathbf{1}_{-1}, \mathbf{g}^{\perp}\right) \neq 0$. It is not known whether $\mathbf{P}_{1} \times \mathbf{P}_{n_{2}} \times \cdots \mathbf{P}_{n_{m}}$ with $2 \leq n_{2}, \ldots n_{m}$ is determined by its fundamental forms. For $m=2$, this was conjectured to be true in [L1, Section $6]$.

\section{References}

[GH] Griffiths, P. and Harris, J.: Algebraic geometry and local differential geometry. Ann. scient. Éc. Norm. Sup. 12 (1979) 35う-432

[JM] Jensen, G. and Musso, E.: Rigidity of hypersurfaces in complex projective space. Ann. scient. Éc. Norm. Sup. 27 (1994) 227-248

[K] Kostant, B.: Lie algebra cohomology and generalized Borel-Weil theorem. Ann. Math. 74 (1961) 329-387

[L1] Landsberg, J. M.: On the infinitesimal rigidity of homogeneous varieties. Compositio Math. 118 (1999) 189-201

[L2] Landsberg, J. M.: Griffiths-Harris rigidity of compact Hermitian symmetric spaces. preprint. alg-geom/0207287.

[SYY] Sasaki, T., Yamaguchi, K. and Yoshida, M.: On the rigidity of differential systems modelled on Hermitian symmetric spaces and disproofs of a conjecture concerning modular interpretations of configuration spaces. Adv. Stud. Pure Math. 25 CR-geometry and overdetermined systems. (1997) 318-354

[S] Se-ashi, Y., On differential invariants of integrable finite type linear differential equations. Hokkaido Math. J. 17 (1988) 151-195

[W] Wilczynski, E. J.: Projective differential geometry of curves and ruled surfaces. reprinted by Chelsca Publ. Co. Teubner, 1906

[Y] Yamaguchi, K.: Differential systems associated with simple graded Lie algebras. Adv. Stud. Pure Math. 22 Progress in differential geometry. (1993) 413-494

Jun-Muk Hwang

Korea Institute for Advanced Study

207-43 Cheongryangri-dong

Scoul 130-012, Korea

e-mail: jmhwang@ins.kias.re.kr
Keizo Yamaguchi

Department of Mathematics, Faculty of Science

Hokkaido Univorsity

Sapporo 060-0810, Japan

e-mail: yamaguch@math.sci.hokudai.ac.jp 